\title{
Tibial and femoral cartilage changes in knee osteoarthritis
}

\author{
F M Cicuttini, A E Wluka, S L Stuckey
}

\begin{abstract}
Background-Despite the increasing interest in using knee cartilage volume as an outcome measure in studies of osteoarthritis $(\mathrm{OA})$, it is unclear what components of knee cartilage will be most useful as markers of structure in the tibiofemoral (TF) joint.

Objective-To compare the changes that occur in femoral and tibial cartilage volume in normal and osteoarthritic knees and how they relate to radiological grade.
\end{abstract}

Methods-82 subjects (44 female, 38 male, age range 35-69 years) with a spectrum of radiological knee OA were examined. Each subject had femoral and tibial cartilage volume in the medial and lateral TF joint determined from $T_{1}$ weighted fat saturated magnetic resonance images of the knee. Radiological grade of OA was determined from standing knee radiographs.

Results-There was strong correlation between femoral and tibial cartilage volume measured in both the medial $(R=0.75, p<0.001)$ and lateral $T F$ joint $(R=0.77, p<0.001)$. Similar correlations persisted when those with normal and those with OA joints were examined separately at both the medial and lateral TF joint. For each increase in radiological grade of joint space narrowing (0-3), there was a mean (SD) reduction in tibial cartilage volume of $1.00(0.32) \mathrm{ml}$ in the medial compartment and $0.53(0.25) \mathrm{ml}$ in the lateral compartment, after adjusting for differences in bone size. Similar changes were seen in the femoral cartilage.

Conclusions-The amounts of tibial and femoral cartilage are strongly related. It may be that for TF joint disease, measuring tibial cartilage alone may be adequate, given that measurements of the total femoral cartilage are less reproducible and the difficulties inherent in identifying the most appropriate component of femoral cartilage to measure.

(Ann Rheum Dis 2001;60:977-980)

There has been increasing interest in the use of magnetic resonance imaging (MRI) in the measurement of knee cartilage volume as a possible outcome measure in arthritis. ${ }^{1-3}$ MRI has been shown to be a valid measure of cartilage volume when MRI cartilage volume is compared with anatomical dissection, and to be reproducible, with coefficient of variations of about $2 \% .^{2-4}$ This technique has been used to explore factors that influence knee cartilage in healthy adults and children. ${ }^{34}$

One potential problem is that most techniques currently in use to measure knee cartilage volume use varying levels of manual manipulation and are consequently quite time consuming. ${ }^{1-3}$ One possible approach is to try to identify whether it is possible to limit the components of knee cartilage being measured and still retain cartilage measures that are a valid measure of the state of joint cartilage. In this study we compared the changes that occur in femoral and tibial cartilage volume in normal and osteoarthritic knees and how they relate to radiological grades.

\section{Methods}

Eighty two subjects ( 44 female, 38 male, age range 35-69 years) with a spectrum of radiological knee osteoarthritis (OA) from normal to grade 3 osteophytes and joint space narrowing were included in this study. Each subject had an MRI and an $x$ ray examination performed with a weightbearing anteroposterior view in extension on the same knee. The patients knees were imaged in the sagittal plane on a $1.5 \mathrm{~T}$ whole body magnetic resonance unit (Sigma Advantage GE Medical Systems Milwaukee, WIS) with use of a commercial transmit-receive extremity coil, as previously described. $^{34}$

Blinded radiographs were scored using a standardised radiographic atlas read on two separate occasions by one investigator. ${ }^{5}$ The intraobserver reliability as measured by $\kappa$ coefficient was 0.87 and 0.92 for joint space narrowing and osteophytes at the tibiofemoral (TF) joint.

REFORMATTING THE ORIGINAL SAGITTAL DATA SET INTO CORONAL PLANE

To transform the images to the coronal plane the Analyse Software package developed by the Mayo Clinic was employed, and the images were resized to an isotropic volume of $256 \times 256 \times 144$ slices with a voxel size of 0.624 $\mathrm{mm}$. This volume was then transformed to the coronal plane and padded to 256 pixels to retain consistent $\mathrm{X} \times \mathrm{Y}$ dimensions. The measurement of all 256 interpolated slices that resulted does not improve accuracy over the original acquisition set of 60 slices, as the interpolated slices do not contain any "new" data. When every third slice was used this resulted in 86 slices of thickness $1.281 \mathrm{~mm}$ and a set size of $256 \times 256 \times 86$ based on a voxel size of $0.427 \mathrm{~mm} \times 0.427 \mathrm{~mm} \times 1.281 \mathrm{~mm}$. The accuracy of the coronal measurements was tested by comparing them with measurements 

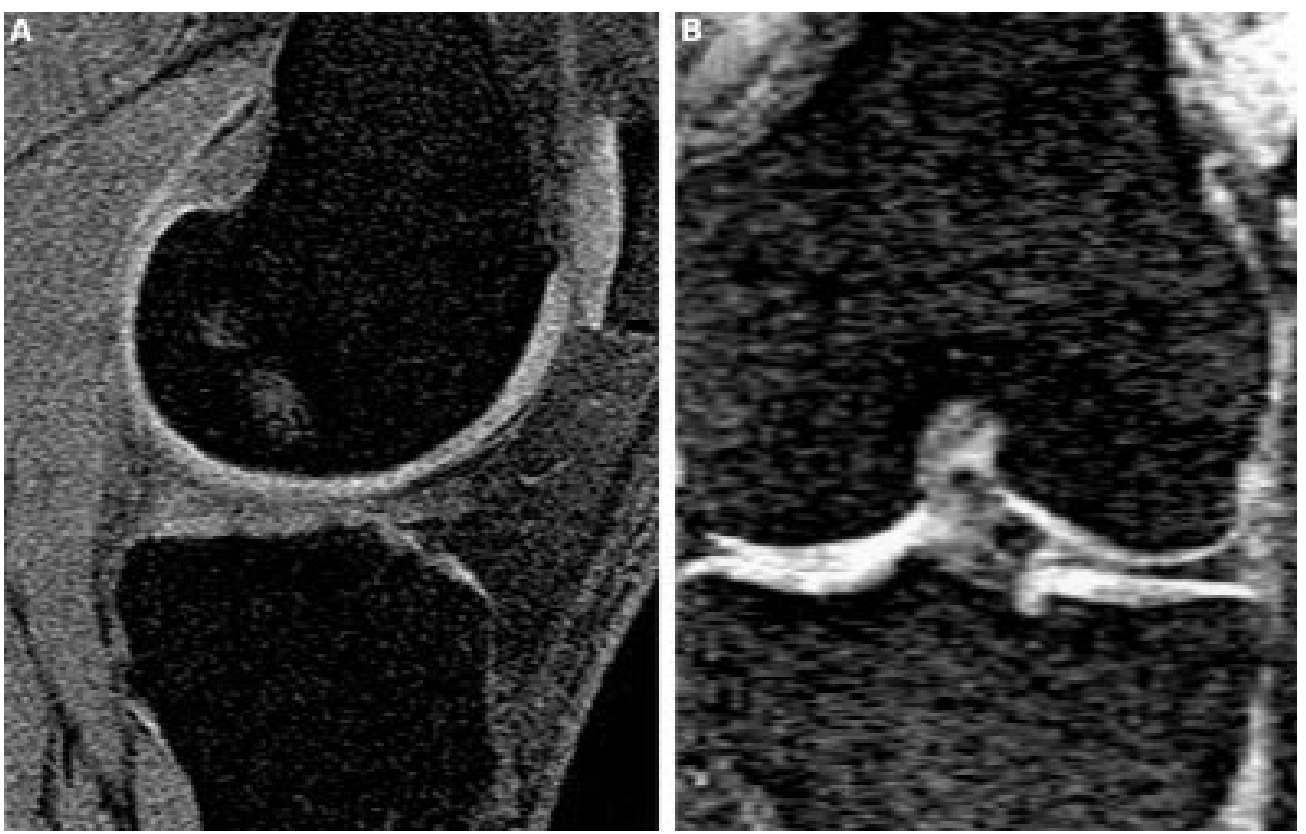

Figure 1 (A) Single $T_{1}$ weighted fat saturation sagittal image of a study subject's knee using spoiled gradient echo imaging. (B) a similar image of the same subject's knee from the coronal image reformation.

from the original sagittal plane, where all 60 slices were measured. The above process was repeated with the images transformed into the axial plane to create a third set of images.

MEASUREMENT OF CARTILAGE VOLUMES AND TIBIAL PLATEAU AREAS

As the femoral cartilage is a continuous structure and forms part of three joints (the patellofemoral and medial and lateral $\mathrm{TF}$ joints), the coronal view was used to measure both the femoral and tibial cartilage, because this sequence allows best visualisation of the femoral cartilage component of the medial and lateral TF joints and is satisfactory for the tibial cartilage (fig 1). Similar tibial cartilage volumes are obtained from the original sagittal sequence and the reformatted coronal data. The average over- and underestimate of lateral and medial tibial cartilage volume from the reformatted coronal scans compared with the originally acquired sagittal sequences was $3.5 \%$ and $3.8 \%$.

Articular cartilage volumes were determined by $3 \mathrm{D}$ image processing on an independent work station using the Osiris Software package (University of Geneva) as previously described. $^{34}$ The intraobserver reproducibility for repeat measures of cartilage volume from single acquisitions were: medial tibial coefficients of variation (CV) $2.3 \%$ and intraclass correlation coefficient (ICC) 0.993; lateral tibia CV 2.4\% and ICC 0.992. For the femoral cartilage volumes, the reproducibility measures were: medial femoral CV $2.6 \%$ and ICC 0.992; for the lateral femoral cartilage volumes, the CV was $2.8 \%$ and ICC 0.988 . The areas of the medial and lateral tibial plateaux were directly measured by manually drawing contours on the reformatted axial data. ${ }^{4}$ The intraobserver reproducibility measures for tibial plateaux were: medial CV 2.7\% and ICC 0.988 ; for the lateral femoral cartilage volume, the $\mathrm{CV}$ was $2.8 \%$ and ICC 0.986 .

\section{DATA ANALYSIS}

Pearson's correlation coefficient was used to examine the association between femoral and tibial cartilage volume at the medial and lateral $\mathrm{TF}$ joint and then radiological grade of OA. Linear regression was used to adjust for tibial condylar area (as a measure of bone size). Results are presented as regression coefficients that represent differences in cartilage volume per unit change in the relevant explanatory factor, while other factors are held constant (that is, controlled for). The adequacy of the regression model was assessed using standard regression diagnostic techniques. ${ }^{6}$

\section{Results}

In the 82 subjects examined in this study, with a mean (SD) age of 43.4 (12.8) years, radiological OA was present in $64(78 \%)$ knees in the medial TF joint and in $40(49 \%)$ knees in the lateral $\mathrm{TF}$ joint.

There was a strong correlation between the amount of femoral and tibial cartilage measured in both the medial and lateral TF joint compartments (table 1). In the medial compartment, the average (SD) volume of the femoral cartilage was $1.59(1.0) \mathrm{ml}$ and of the tibial cartilage $1.54(0.74) \mathrm{ml}(\mathrm{p}=0.21$ for difference) with a correlation of $0.75(\mathrm{p}<0.001)$. In the lateral compartment, the average volume of the femoral cartilage was $1.91(1.07) \mathrm{ml}$ and of the tibial cartilage $1.73(0.77) \mathrm{ml}(\mathrm{p}=0.11$ for difference) with a correlation of 0.77 $(\mathrm{p}<0.001)$. When those with normal knees and those with radiological OA were examined separately, similar results were found. In the medial TF joint, the correlation between the femoral and tibial cartilage in those with normal knees was $0.70(p=0.009)$ and in those 
Table 1 Femoral and tibial cartilage volumes in the tibiofemoral joints

\begin{tabular}{|c|c|c|c|}
\hline & $\begin{array}{l}\text { Femoral } \\
\text { Mean (SD) } m l\end{array}$ & $\begin{array}{l}\text { Tibial } \\
\text { Mean (SD) } m l\end{array}$ & Correlation $(R)$ \\
\hline \multicolumn{4}{|l|}{ Medial tibiofemoral (TF) joint } \\
\hline All subjects $(n=82)$ & $1.59(1.0)$ & $1.54(0.74)$ & $0.75(\mathrm{p}<0.001)$ \\
\hline Subjects with no medial TF OA* $(n=18)$ & $2.07(0.88)$ & $1.76(0.63)$ & $0.70(\mathrm{p}=0.009)$ \\
\hline Subjects with medial TF OA $(n=64)$ & $1.42(1.00)$ & $1.46(0.77)$ & $0.75(\mathrm{p}<0.001)$ \\
\hline \multicolumn{4}{|c|}{ Subjects with different grades of medial $\mathrm{TF}$ joint space narrowing: } \\
\hline Grade $=0(n=17)$ & $2.27(0.84)$ & $1.86(0.60)$ & $0.79(\mathrm{p}<0.001)$ \\
\hline Grade $=1(n=26)$ & $1.63(0.98)$ & $1.52(0.89)$ & $0.63(\mathrm{p}=0.016)$ \\
\hline Grade $=2(n=24)$ & $0.97(0.73)$ & $1.38(0.65)$ & $0.88(\mathrm{p}=0.01)$ \\
\hline Grade $=3(n=14)$ & $0.69(0.68)$ & $0.74(0.42)$ & $0.98(\mathrm{p}=0.014)$ \\
\hline \multicolumn{4}{|l|}{ Lateral tibiofemoral joint } \\
\hline All subjects $(n=82)$ & $1.91(1.07)$ & $1.73(0.77)$ & $0.77(\mathrm{p}<0.001)$ \\
\hline Subjects with no lateral TF OA $(n=40)$ & $2.10(1.14)$ & $1.81(0.72)$ & $0.80(\mathrm{p}<0.001)$ \\
\hline Subjects with lateral TF OA $(n=42)$ & $1.71(0.99)$ & $1.63(0.83)$ & $0.75(\mathrm{p}<0.001)$ \\
\hline \multicolumn{4}{|c|}{ Subjects with different grades of lateral TF joint space narrowingt: } \\
\hline Grade $=0(n=39)$ & $1.85(0.73)$ & $2.00(1.00)$ & $0.74(\mathrm{p}<0.001)$ \\
\hline Grade $=1(n=24)$ & $1.54(0.86)$ & $1.44(1.07)$ & $0.88(\mathrm{p}<0.000)$ \\
\hline Grade $=2(n=14)$ & $1.24(1.12)$ & $1.15(0.74)$ & $0.84(\mathrm{p}=0.01)$ \\
\hline
\end{tabular}

${ }^{\star} \mathrm{OA}$ is defined as the presence of osteophytes or joint space narrowing score of $\geqslant 1$, or both.

†Only five subjects had grade 3 lateral joint space narrowing, so no data are presented.

with radiological OA, $0.75(\mathrm{p}<0.001)$. In the lateral $\mathrm{TF}$ joint, the correlation between the femoral and tibial cartilage in those with normal knees was $0.80(\mathrm{p}<0.001)$ and in those with radiological OA $0.75(\mathrm{p}<0.001)$.

Knee cartilage was reduced in those with OA (table 1). Parallel changes were seen in the femoral and tibial cartilages in both the medial and lateral TF compartments as the grade of joint space narrowing increased. In the medial $\mathrm{TF}$ joint, for each increase in radiological grade of joint space narrowing (0-3), the femoral cartilage decreased by $1.86(0.33) \mathrm{ml}$ while the tibial cartilage was reduced by $1.00(0.32) \mathrm{ml}$, after adjusting for differences in bone size as measured by medial tibial bone area. In the lateral TF joint, for each increase in radiological grade of joint space narrowing $(0-3)$ the femoral cartilage decreased by $0.62(0.30) \mathrm{ml}$ while the tibial cartilage decreased by $0.53(0.25) \mathrm{ml}$, after adjusting for differences in bone size as measured by lateral tibial bone area.

\section{Discussion}

In this study we have shown that there is a strong correlation between femoral and tibial cartilage volume in the medial and lateral TF joints both in subjects with normal knees and in those with radiological OA. Knee cartilage volume correlated inversely with radiological grade of joint space narrowing.

The femoral cartilage is a continuous structure that forms part of three joints- the patellofemoral joint, the medial and lateral TF joints. There is no clearly defined anatomical boundary between the femoral cartilage component of each of these three joints. In most epidemiological studies, joint space narrowing is used as a surrogate measure for joint cartilage and is the recommended outcome measure for anatomical progression of disease. ${ }^{7}$ However, this measure is not sensitive to small changes, and it is well recognised that noncartilage structures, such as the menisci, may be included in this measurement. ${ }^{8}$ Increasingly, cartilage volume as measured by MRI is being investigated. ${ }^{910}$ However, it is still unclear which components of knee cartilage are the most useful to measure and the most efficient, given that most methods currently in use to measure knee cartilage volume are time consuming as they require varying degrees of manual processing. ${ }^{1-3}$

This study suggests that similar information about the structure of the lateral and medial $\mathrm{TF}$ joint can be obtained by measuring either the femoral or tibial cartilages. There was a strong correlation between the femoral cartilage, as measured from the coronal sequence, and the tibial cartilage in both medial and lateral $\mathrm{TF}$ joints in normal subjects and in those with OA. There was a strong, inverse relation between the radiological grade of joint space narrowing in both TF joints and in both femoral and tibial cartilage volume.

A potential problem in our study is that the original MRI images were acquired in the sagittal plane. However, from this sequence it is difficult to identify reproducibly the femoral cartilage that would correspond to the TF joint as seen on standing knee radiographs. For this reason, we reformatted the data into the coronal plane. Reconstruction of MRI images into different planes has been widely used clinically. ${ }^{11-14}$ We found that similar tibial cartilage volumes are obtained from the original sagittal sequence and the reformatted coronal data, with the average over- and underestimate of lateral and medial tibial cartilage volume from the reformatted coronal scans being 3.5\% and 3.8\% compared with the originally acquired sagittal sequences. Although sagitally acquired images give a good definition of the tibial cartilage, to avoid any differences introduced by reformatting we compared the tibial and femoral cartilage volumes, both calculated from the reformatted coronal MRI data. Possibly, large osteophytes may exaggerate the apparent cartilage loss where we adjusted for tibial condylar size. However, on axial images, it is possible to visualise the articular surface and measure this, which minimises this potential problem.

Although the technique for measuring both femoral and tibial cartilages has been validated, ${ }^{1-3}$ these cartilages have only been examined in isolation. There has been no examination of the relation between femoral and tibial cartilage volume. The relation between cartilage volume and radiological grade of OA has also not previously been examined. Whether cartilage can be used as an interim marker for OA has yet to be determined, though early data suggest that this will be the case. ${ }^{910}$ In cross sectional studies it seems likely that measuring the tibial cartilage only, may be adequate for TF disease. This has advantages because measurements of the total femoral cartilage are less reproducible and there are difficulties inherent in identifying the most appropriate component of femoral cartilage to measure. ${ }^{1-3}$ We believe that use of the tibial cartilage has an advantage in that it is a clearly defined, anatomical structure and thus less susceptible to error in its identification across different study sites and in different operator's hands. However, the sensitivity and specificity of the femoral and tibial cartilages for detecting change over time will need to be tested in a longitudinal study. In the case of studies that examine cartilage defects, which 
may not necessarily be symmetrical across the joint, measurement of both femoral and tibial cartilage may be required.

This study was supported by the National Health and Medical Research Council. Special thanks to the people who participated and made this study possible.

1 Peterfy CG, van Dijke CF, Janzen DL, Gluer CC, Namba R, Majundar S, et al. Quantification of articular cartilage in the knee with pulsed saturation transfer subtraction and fat-suppressed MR imaging: optimization and validation. Radiology 1994;192:485-91.

2 Eckstein F, Westhoff J, Sittek H, Maag K-P, Haubner M, Faber $\mathrm{H}$, et al. In vivo reproducibility of three-dimensional cartilage volume and thickness measurements with MR imaging. AJR Am J Roentgenol 1998;170:593-7.

3 Cicuttini F, Forbes A, Morris K, Darling S, Bailey M, Stuckey S. Gender differences in knee cartilage volume as measured by magnetic resonance imaging. Osteoarthritis measured by magnetic resor

4 Jones G, Glisson M, Hynes K, Cicuttini F. Gender and site differences in cartilage development: a possible explanation for variations in knee osteoarthritis in later life. Arthritis Rheum 2000;43:2543-9.

5 Burnett S, Hart DJ, Cooper C, Spector TD. A radiographic atlas of osteoarthritis. London: Springer, 1994

6 Neter J, Wasserman W, Kutner M. Applied linear statistical models. 3rd ed. New York: Irwin Publishing, 1990:chapter 11
7 Lequesne M, Brandt K, Bellamy N, Moskowitz R, Menkes $\mathrm{C}$, Pelleiier J-P. Guidelines for testing slow acting drugs in osteoarthritis. J Rheumatol 1994;21 (suppl 41):65-73.

8 Adams JG, McAlindon TE, Dimasi M, Carey J, Eustance S. Contribution of meniscal extrusion and cartilage loss to joint space narrowing in osteoarthritis. Clin Radiol 1999;54:502-6.

9 Peterfy CG, White DL, Zhao J, Van Dijke CF, Genant HK. Longitudinal measuement of knee articular cartilage volume in OA. Arthritis Rheum 1998;41:PS361.

10 Raynauld J-P, Kauffmann C, Godbout B, Beaudoin G, Berthiaume M-J, DeGuise J, et al. Knee osteoarthritis progression evaluated by magnetic resonance imaging and a novel quantification software tool [abstract]. Arthritis Rheum 2000;43(suppl):S399.

11 Sugimoto $\mathrm{H}$, Hyodoh $\mathrm{K}$, Shinozaki T. Throwing injury of the elbow: assessment with gradient three-dimensional, fourier transform gradient-echo and short tau inversion recovery images. J Magn Reson Imaging 1998;8:487-92.

12 Rubin DA, Kneeland JB, Kitay GS, Naranja RJ Jr. Flexor tendon tears in the hand: use of MR imaging to diagnose degree of injury in a cadaver model. AJR Am J Roentgenol 1996;166:615-20.

13 Sisodiya SM, Free SL, Fish DR, Shorvon SD. Increasing the yield from volumetric MRI in patients with epilepsy. Magn Reson Imaging 1995;13:1147-52.

14 Traill MR, Kaufman DM, Runge VM, McCarthy JC, Wood ML, Sterns DM, et al. Anatomy of the proximal femur as seen with three-dimensional magnetic resonance imaging. J Arthroplasty 1989;4:361-7. 